\title{
Intravascular Ultrasound-Guided Management of Proximal Left Anterior Descending Artery Aneurysm With Covered Stent - A Case Report
}

\author{
Gurkirat Singh ${ }^{\mathrm{a}, \mathrm{b}}$, Hemant Khemani ${ }^{\mathrm{a}}$, Rahul Singla ${ }^{\mathrm{a}}$, Shakil Shaikh ${ }^{\mathrm{a}}$, \\ Vishal Patil ${ }^{\mathrm{a}}$, Narender Omprakash Bansal ${ }^{\mathrm{a}}$
}

\begin{abstract}
Coronary artery aneurysm is defined as the localized dilatation of a coronary artery segment more than 1.5 times the size of adjacent normal segments. The aneurysms of the coronary arteries are rare. Coronary aneurysms can be congenital or acquired. The majority are atherosclerotic in origin. The primary complication is myocardial ischemia or infarction, sudden cardiac death with rupture being rare. Some aneurysms are diagnosed incidentally in arteries other than the culprit artery. Treatment options include medical management with anticoagulation, percutaneous intervention with covered stents or surgery. We report a case of 67-year-old male who presented with acute coronary syndrome. Coronary angiogram showed a moderate size aneurysm of the proximal left anterior descending artery. This aneurysm was successfully managed percutaneously with a covered stent.
\end{abstract}

Keywords: Intravascular ultrasound; Aneurysm; Covered stent; Acute coronary syndrome

\section{Introduction}

Coronary artery aneurysm (CAA) is defined as the localized dilatation of a coronary artery segment more than 1.5 times the size of adjacent normal segments. Coronary artery ectasia is defined as more diffuse dilatation of coronary artery [1]. CAAs are further divided to saccular aneurysms if the transverse diameter exceeds the longitudinal diameter, and to fusiform aneurysms in the opposite case. CAAs are termed giant if their diameter exceeds the reference vessel diameter by $>4$ times or if they are $>8 \mathrm{~mm}$ in diameter [2]. CAAs are also considered as giant if diameter is $>2 \mathrm{~cm}$. The aneurysms of the coronary

Manuscript submitted July 27, 2018, accepted September 4, 2018

aDepartment of Cardiology, Grant Medical College and Sir J.J. Group of Hospitals, Mumbai, India

${ }^{b}$ Corresponding Author: Gurkirat Singh, Department of Cardiology, 4th Floor, Main Hospital Building, J.J. Hospital Campus, Byculla, Mumbai 400008, India. Email: singhgs269@yahoo.com

doi: https://doi.org/10.14740/cr764w arteries are rare with an estimated prevalence rate of $0.15-5 \%$ in the patients undergoing coronary angiography in different case series [3]. The right coronary artery is the most commonly involved artery followed by left anterior descending artery followed by the left circumflex artery. Left main involvement is very rare [3]. Associated aneurysms of ascending and descending aorta are also described. Complications include thrombosis, rupture or embolism. Clinical presentations range from an incidental finding on cardiac imaging to acute coronary syndrome. Treatment options include medical management with anticoagulation, percutaneous intervention with covered stents or surgery. Due to the lack of trials and guidelines for management of CAAs, treatment of these patients is challenging. We report a case of 67-year-old male who presented with acute coronary syndrome. Coronary angiogram showed a moderate size aneurysm of the proximal left anterior descending artery. This aneurysm was successfully managed percutaneously with a covered stent. He also had a dilated ascending aorta measuring $5.2 \mathrm{~cm}$.

\section{Case Report}

A 67-year-old male patient was admitted with acute onset of chest pain at rest, radiating to left arm, associated with sweating since $12 \mathrm{~h}$. He is a known case of hypertension since 15 years, dyslipidemia since 10 years and chronic smoker since 30 years. He gives a history of similar chest pain 6 months back for which he was admitted in other hospital. He was admitted for 3 days at that time, and no intervention was done as he had some financial problems. On admission, pulse was 66/min; blood pressure was 160/100 mm of mercury. Cardiac examination was normal; respiratory system examination was normal. Qualitative troponin $\mathrm{T}$ was positive. Electrocardiogram (ECG) on admission is shown in Figure 1. Echocardiography showed hypokinesia in basal, mid, distal anteroseptal, anterior and anterolateral segments with ejection fraction of $30 \%$. Routine blood investigations were normal, as shown in Table 1 . He was taken for coronary angiography. Coronary angiography showed (Figs. 2 - 4) CAA in proximal left anterior descending artery (LAD) followed by significant lesion. Proximal OM2 and proximal OM3 showed $99 \%$ and $90 \%$ lesion, respectively. Right coronary artery (RCA) was $100 \%$ occluded after the conal branch retrogradely filling from the left system. 


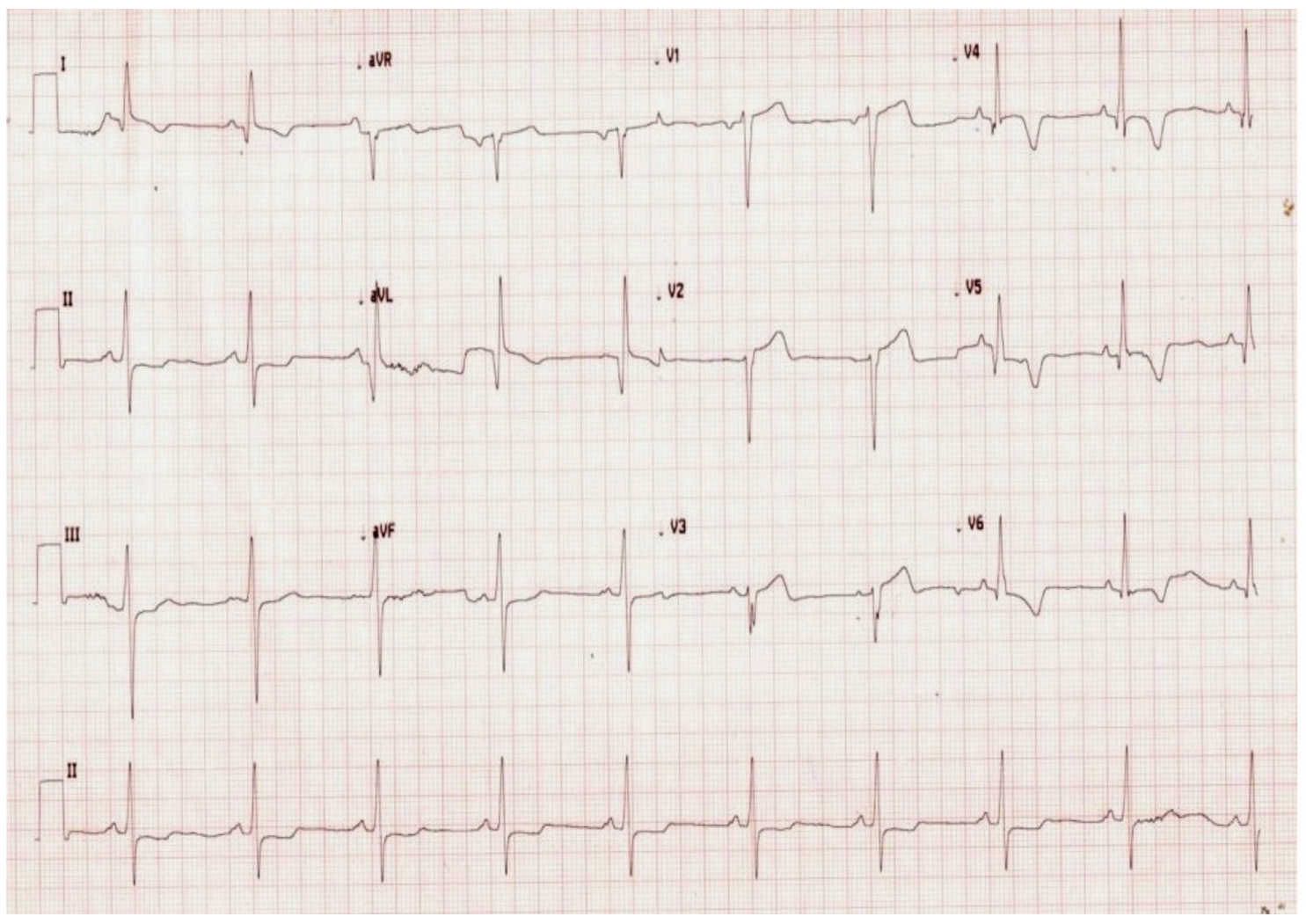

Figure 1. ECG on admission showing $T$ inversion in lead V3-6, I, aVL with loss of $R$ wave in anterior leads.

Ascending aorta was dilated measuring $5.2 \mathrm{~cm}$ (Fig. 5). The options of coronary artery bypass grafting (CABG) and multivessel angioplasty were discussed with patient and relatives. As the patient was not willing for $\mathrm{CABG}$, he was taken for angioplasty.

Left coronary ostium was engaged with JL 7F 5.0 guiding catheter. Obtuse marginal 3 was stented with $3 \times 23 \mathrm{~mm}$ drug-eluting stent (DES) after pre-dilatation. Obtuse marginal 2 was stented with $2.75 \times 21$ DES after pre-dilatation. Intravascular ultrasound (IVUS) was done for LAD to determine whether LAD ostium had a significant lesion. IVUS to LAD showed aneurysm of $7.5 \times 6.5 \mathrm{~mm}$ and $11 \mathrm{~mm}$ in length (Fig. 6). Ostium of LAD was not involved. Lesion in LAD, distal to aneurysm was stented with $3.0 \times 24 \mathrm{DES}$. Graftmaster $3.5 \times 19$ stent was placed across the aneurysm and deployed. Graftmaster is a covered stent consisting of expandable polytetrafluoroethylene (ePTFE) sandwiched between two identical stain- less steel stents. Post-stent dilatation was done with $3.5 \times 13$ NC balloon. Check shoot showed good result (Fig. 7). IVUS showed adequate apposition of the stent with complete exclusion of the aneurysm (Fig. 8). The post-procedure course was uneventful and the patient was shifted to ward after $24 \mathrm{~h}$ of ICCU stay (Fig. 9). Patient was discharged after 5 days.

\section{Discussion}

Morgagni described the first case of coronary artery aneurysm in 1761 [4]. The presence of CAA was first recognized in pathological studies. Munker et al reported the first ante-mortem case diagnosed by coronary angiography in 1958 [5]. Markis et al used the term "ectasia" to describe coronary aneurysmal dilatation and attempted to further classify it on the basis of appearance and the number of coronary arteries involved in

Table 1. Blood Investigations Before and After the Procedure

\begin{tabular}{llll}
\hline Lab parameter & Reference range & Before procedure & After procedure \\
\hline Hemoglobin & $13-17 \mathrm{gm} / \mathrm{dL}$ & $13.4 \mathrm{gm} / \mathrm{dL}$ & $13.1 \mathrm{gm} / \mathrm{dL}$ \\
Total white blood cell count & $4 \times 10^{9}-11 \times 10^{9} / \mathrm{L}$ & $9.2 \times 10^{9} / \mathrm{L}$ & $10.2 \times 10^{9} / \mathrm{L}$ \\
Platelet count & $150 \times 10^{9}-400 \times 10^{9} / \mathrm{L}$ & $236 \times 10^{9} / \mathrm{L}$ & $228 \times 10^{9} / \mathrm{L}$ \\
Serum creatinine & $0.8-1.4 \mathrm{mg} / \mathrm{dl}$ & $1.1 \mathrm{mg} / \mathrm{dL}$ & $1.2 \mathrm{mg} / \mathrm{dL}$ \\
Sodium/potassium & $135-145 \mathrm{and} 3.5-4.9 \mathrm{mmol} / \mathrm{L}$ & $136 \mathrm{and} 4.4 \mathrm{mmol} / \mathrm{L}$ & $133 \mathrm{and} 4.2 \mathrm{mmol} / \mathrm{L}$ \\
LDL & $<3.0 \mathrm{mmol} / \mathrm{L}$ & $3.4 \mathrm{mmol} / \mathrm{L}$ & \\
\hline
\end{tabular}




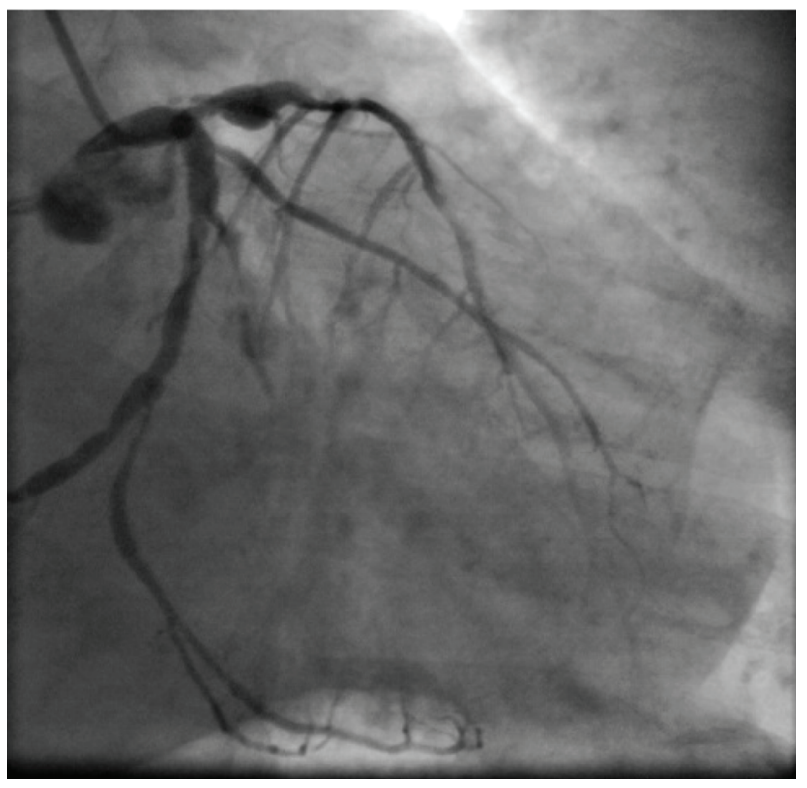

Figure 2. Coronary angiogram in RAO caudal view. OM2 and OM3 has significant lesion. Localized dilatation is noted in proximal LAD.

coronary angiography. They described type 1 as diffuse ectasia of all three major vessels, type 2 as diffuse ectasia in one vessel and localized disease in one other major vessel, type 3 as diffuse ectasia in one vessel only and type 4 as localized segmental ectasia of one vessel [6]. Type 4 is most common and was later recognized as CAA.

CAAs can be congenital or acquired. The most common cause of CAAs is atherosclerosis, but other causes include congenital heart disease, trauma, Kawasaki disease, Marfan syndrome, Ehlers-Danlos syndrome polyarteritis nodosa, Ta-

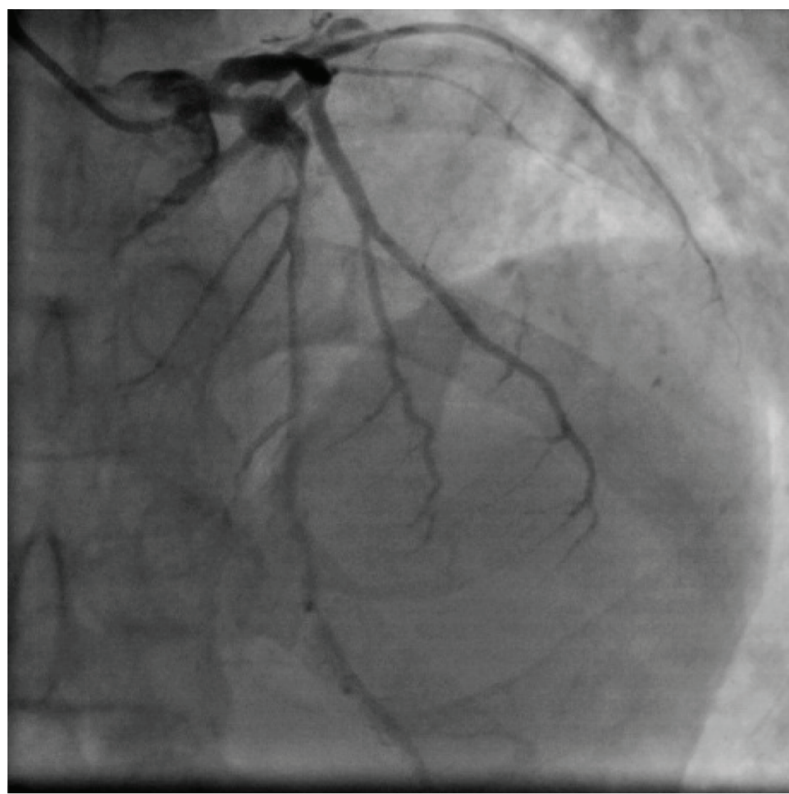

Figure 3. Coronary angiogram AP cranial view. Localized dilatation is seen in proximal LAD followed by significant lesion.

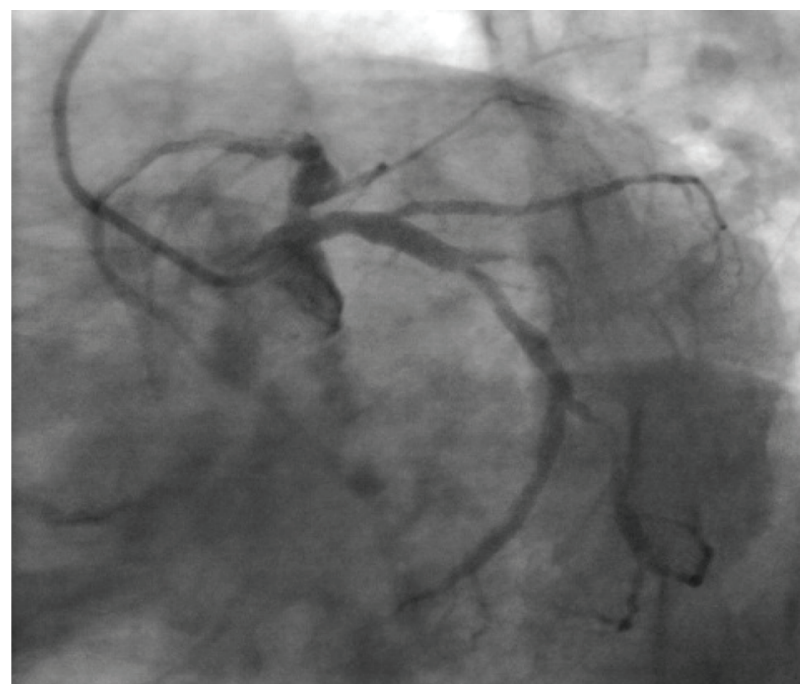

Figure 4. Coronary angiogram LAO caudal view. Short left main artery with localized dilatation in proximal LAD. Left circumflex artery ostium showed no significant lesion.

kayasu arteritis, syphilitic aortitis, scleroderma, systemic lupus erythematosus, Behcet disease and fibromuscular dysplasia [7]. CAAs have been reported as a result of percutaneous coronary interventions mostly after coronary angioplasties. Various causative factors have been postulated including residual nonhealing dissection, arterial barotrauma due to high-pressure balloon inflation, and the use of oversized balloons or stents, atherectomy techniques and laser angioplasty [8]. Antiproliferative DES can cause CAAs by mechanisms, such as delayed re-endothelialization, inflammatory changes of the medial wall and hypersensitivity reactions [9]. In our patient, the cause of aneurysm was atherosclerosis in view of the risk factor profile and associated significant lesions in other coronary arteries.

Most CAAs are asymptomatic and are only detected in-

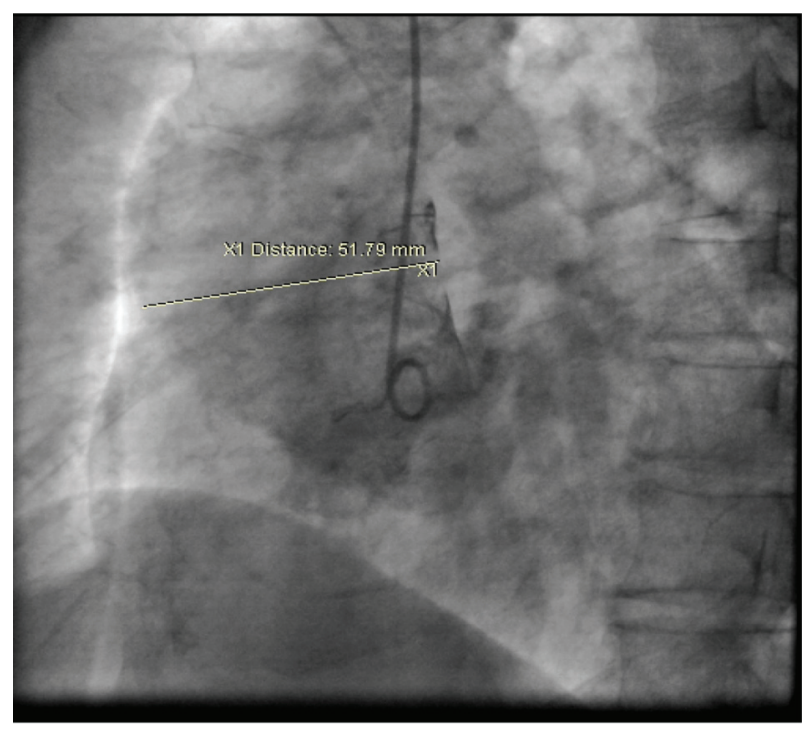

Figure 5. Aortic root-shoot in LAO view. The ascending aorta is dilated measuring $5.2 \mathrm{~cm}$. 


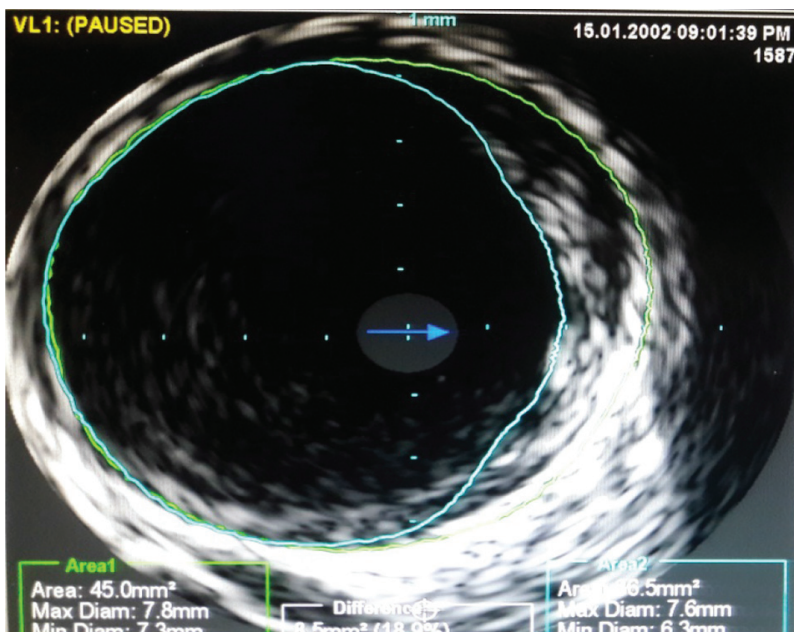

Figure 6. Intravascular ultrasound (IVUS) to LAD. Localized dilatation of $7.5 \times 6.5 \mathrm{~mm}$ was seen in proximal LAD.

cidentally during coronary angiography or computed tomography. However, some patients present with angina pectoris, myocardial infarction or sudden cardiac death. The presence of concomitant obstructive atherosclerotic disease can result in effort angina or acute coronary syndrome. Local thrombosis in the lumen of large aneurysms may lead to distal embolization and myocardial infarction. Massive enlargement of some CAAs can result in compression of adjacent structures like superior vena cava. Rupture of aneurysm can cause acute cardiac tamponade or sudden cardiac death [10].

Coronary angiography remains the gold standard for the diagnosis of ectatic or aneurismal coronary arteries.

IVUS can also accurately size the CAA and/or any adjacent stenoses and allows proper stent sizing if percutaneous

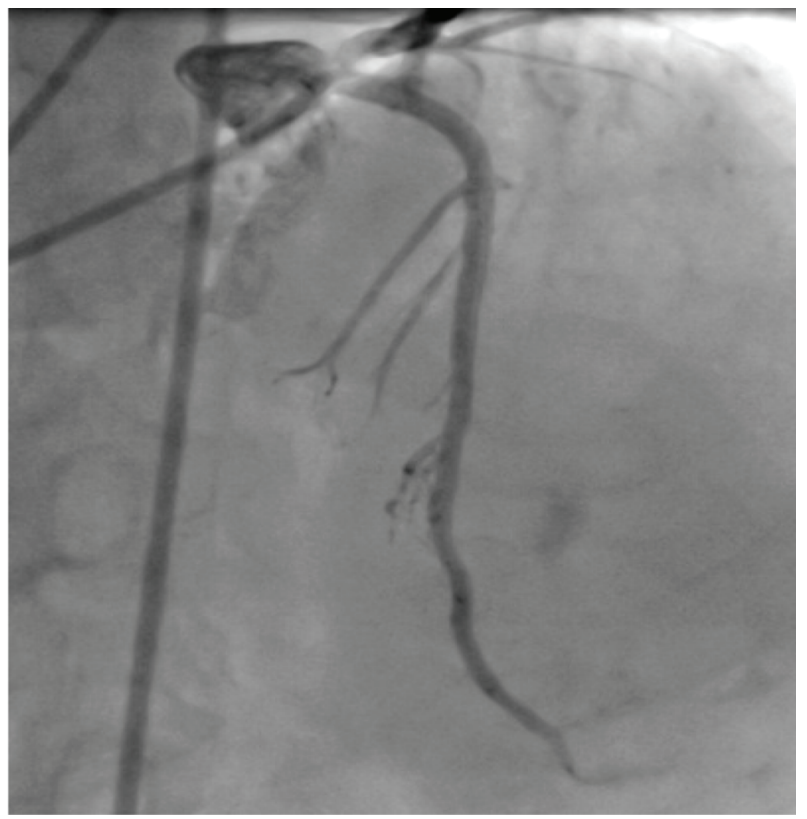

Figure 7. Coronary angiogram in AP cranial view after stenting showing good result with complete exclusion of aneurysm.

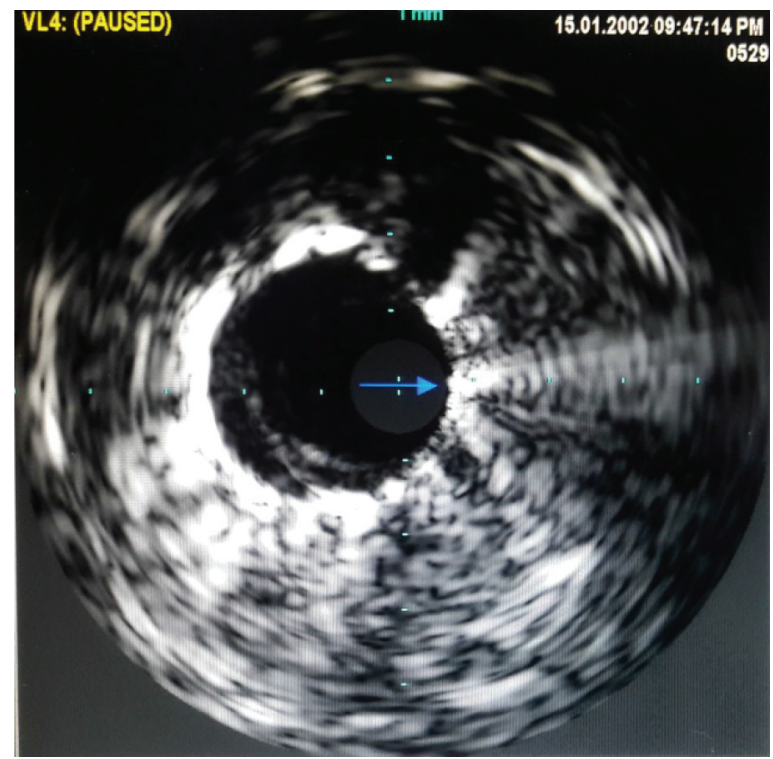

Figure 8. Intravascular ultrasound after implantation of Graftmaster stent in proximal LAD across aneurysm showing adequate apposition of the stent and complete exclusion of aneurysm.

intervention is planned. At present, there is no consensus on the optimal management of CAAs, and the treatment options for CAAs include surgical, percutaneous and medical approaches. Most of the current recommendations are based on small case series or anecdotal evidence. The treatment strategies should be individualized based on the location and morphology of the CAA, the patient's characteristics and the clinical presentation. The largest experience in adults has been with surgical management, which typically includes bypass grafting, aneurysm ligation or resection. Medical therapy includes risk factor modification, as atherosclerosis is the most common cause, dual antiplatelets and anticoagulation in selected patients [11].

Percutaneous techniques, which include exclusion with a covered stent or coil embolization, are used in selected patients. These techniques are reasonable for aneurysms less than $10 \mathrm{~mm}$ [12-14]. For diameters between 2.75 and $5 \mathrm{~mm}$, the Graftmaster coronary stent graft is mostly used. If a larger diameter stent is needed $(5-10 \mathrm{~mm})$, the atrium iCAST balloon expandable covered stent is used to treat CAA. The Graftmaster stent is constructed using a sandwich technique, whereby an ultra-thin layer of ePTFE is placed between two stainless steel stents, which are then pre-mounted on a balloon catheter delivery system. The new PK papyrus-covered stent has greater bending flexibility and a smaller crossing profile compared with the Graftmaster stent. In our patient, Graftmaster $3.5 \times 19$ $\mathrm{mm}$ stent was used for the CAA of proximal LAD [15].

\section{Conclusions}

With the increase in angiography and the more widespread use of high-resolution CT scans and MRI, the diagnosis of coronary aneurysms is expected to become more frequent. We 


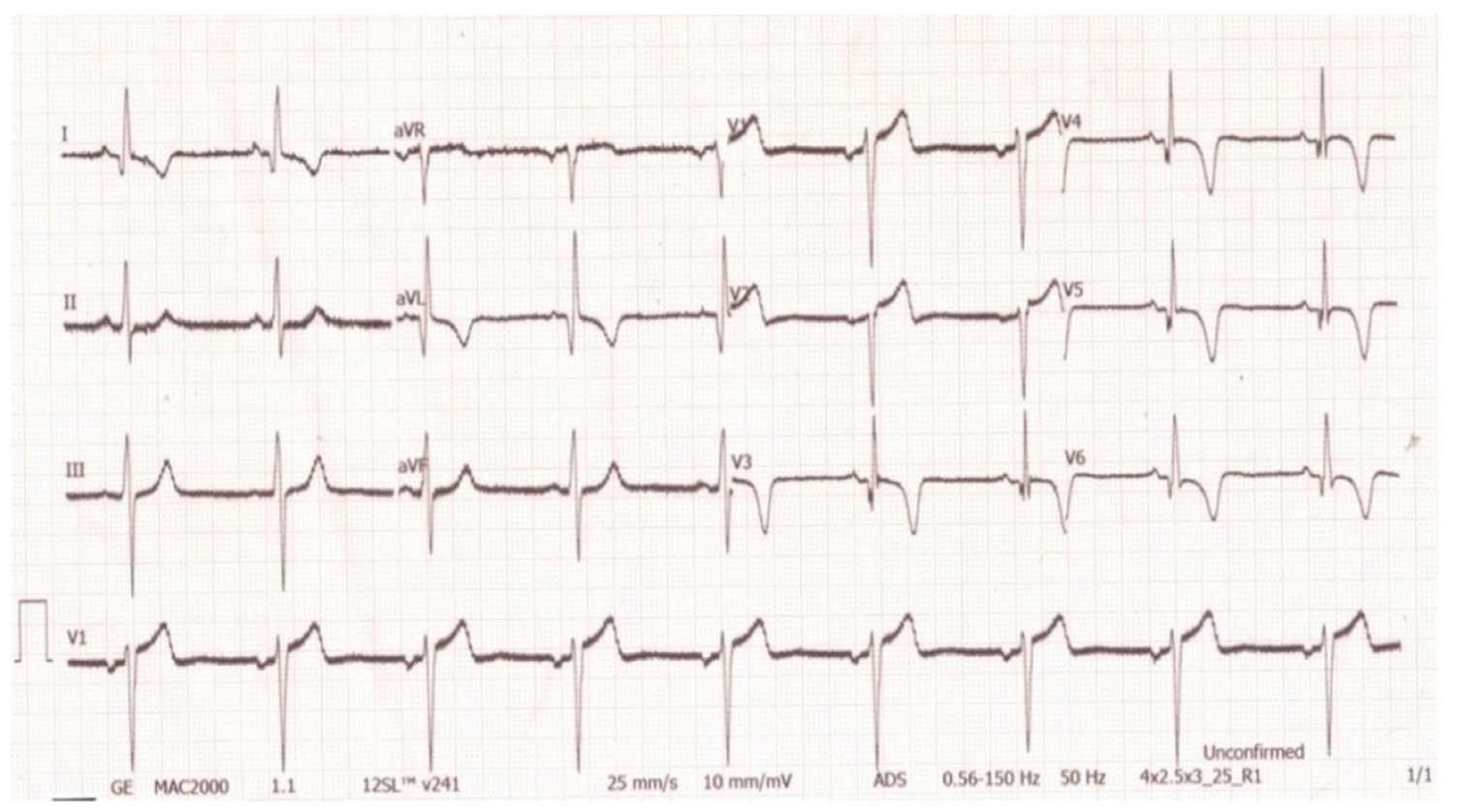

Figure 9. ECG post procedure showing no fresh changes.

need robust evidence-based management strategies. The management of CAAs presents a clinical challenge and should be individualized based on a comprehensive clinical evaluation that encompasses the patient's cardiovascular risk factors, comorbidities, and the nature and anatomy of the CAA. Percutaneous intervention with the exclusion of aneurismal lumen by the placement of covered stent is a feasible and safe procedure even in patients presenting with acute coronary syndrome.

\section{Acknowledgments}

We are grateful to Dr Narender Omprakash Bansal, Professor and Head of Department of Cardiology, Grant Medical College and Sir J.J. Group of Hospital, Mumbai for allowing us to publish this case report.

\section{Conflict of Interest}

None.

\section{References}

1. Luo Y, Tang J, Liu X, Qiu J, Ye Z, Lai Y, Yao Y, et al. Coronary artery aneurysm differs from coronary artery ectasia: angiographic characteristics and cardiovascular risk factor analysis in patients referred for coronary angiography. Angiology. 2017;68(9):823-830.

2. Dajani AS, Taubert KA, Takahashi M, Bierman FZ, Freed MD, Ferrieri P, Gerber M, et al. Guidelines for long-term management of patients with Kawasaki disease. Report from the Committee on Rheumatic Fever, Endocarditis, and Kawasaki Disease, Council on Cardiovascular Disease in the Young, American Heart Association. Circulation. 1994;89(2):916-922.

3. Syed M, Lesch M. Coronary artery aneurysm: a review. Prog Cardiovasc Dis. 1997;40(1):77-84.

4. Morgagni JB. The seats and causes of diseases investigated by anatomy. Venice, Italy: Ex typographia Remondiniana. 1761;27:28.

5. Munkner T, Petersen O, Vesterdal J. Congenital aneurysm of the coronary artery with an arteriovenous fistula. Acta radiol. 1958;50(4):333-340.

6. Markis JE, Joffe CD, Cohn PF, Feen DJ, Herman MV, Gorlin R. Clinical significance of coronary arterial ectasia. Am J Cardiol. 1976;37(2):217-222.

7. Cohen P, O'Gara PT. Coronary artery aneurysms: a review of the natural history, pathophysiology, and management. Cardiol Rev. 2008;16(6):301-304.

8. Bal ET, Thijs Plokker HW, van den Berg EM, Ernst SM, Gijs Mast E, Gin RM, Ascoop CA. Predictability and prognosis of PTCA-induced coronary artery aneurysms. Cathet Cardiovasc Diagn. 1991;22(2):85-88.

9. Aoki J, Kirtane A, Leon MB, Dangas G. Coronary artery aneurysms after drug-eluting stent implantation. JACC Cardiovasc Interv. 2008;1(1):14-21.

10. Rath S, Har-Zahav Y, Battler A, Agranat O, Rotstein Z, Rabinowitz B, Neufeld HN. Fate of nonobstructive aneurysmatic coronary artery disease: angiographic and clinical follow-up report. Am Heart J. 1985;109(4):785-791.

11. Pahlavan PS, Niroomand F. Coronary artery aneurysm: a review. Clin Cardiol. 2006;29(10):439-443.

12. Ohtsuka M, Uchida E, Yamaguchi H, Nakajima T, Akazawa H, Funabashi N, Kobayashi Y, et al. Coronary aneurysm reduced after coronary stenting. Int J Cardiol. 2007;121(1):76-77. 
13. Kilic ID, Fabris E, Serdoz R, Caiazzo G, Foin N, AbouSherif S, Di Mario C. Coronary covered stents. EuroIntervention. 2016;12(10):1288-1295.

14. Briguori C, Sarais C, Sivieri G, Takagi T, Di Mario C, Colombo A. Polytetrafluoroethylene-covered stent and coronary artery aneurysms. Catheter Cardiovasc Interv. 2002;55(3):326-330.

15. Kawsara A, Nunez Gil IJ, Alqahtani F, Moreland J, Rihal CS, Alkhouli M. Management of coronary artery aneurysms. JACC Cardiovasc Interv. 2018;11(13):1211-1223. 